\title{
Bewegung für Menschen mit Demenz
}

\author{
Angehende Gesundheits- und Pflegemanager der FH Kärnten starten Initiative
}

Die Studiengänge Gesundheits- und Pflegemanagement der Fachhochschule Kärnten haben sich einer anwendungsorientierten, praxisnahen Ausbildung verschrieben. Ganz in diesem Sinne entwickelten drittsemestrige Bachelorstudierende im Wintersemester eine Kampagne, die auf die Bedeutsamkeit von Bewegung für Menschen mit Demenz hinweist. Im Zuge der Lehrveranstaltung "Anwendungsprojekt Gesundheitsförderung" widmeten sich die Studierenden unter Leitung von Prof. (FH) Eva Mir dem Themenkomplex Bewegungsförderung und Demenz.

Ausgangspunkt war die wissenschaftlich belegte Tatsache, dass sich ein bewegtes Leben positiv auf die Erkrankung Demenz auswirkt. „Wir wissen aus zahlreichen Studien, dass Bewegung bei Menschen mit Demenz nicht nur körperliche Aspekte wie etwa Bewegungsfähigkeit, Muskelkraft, Gleichgewicht oder Stabilität beim Stehen fördert, sondern sich auch positiv auf kognitive Fähigkeiten auswirkt," erklärt Mir. Dadurch kann das Fortschreiten demenzieller Erkrankungen mittels Bewegung verlangsamt werden. Diese Argumente gilt es aber an Betroffene, Angehörige und Personen des Gesundheitswesens zu vermitteln. Genau an dieser Stelle setzt die Kampagne „Demenz. Bewegt.Leben" an.

\section{Aufklärungs- und \\ Sensibilisierungsmaterialien}

Die Studierenden erstellten ein Plakat und Postkarten, die unterschiedliche Bewegungsarten darstellen, ein Informationsblatt für Angehörige zum Thema Bewegungsförderung bei Menschen mit Demenz sowie ein Video, das auch einen Beitrag zur Entstigmatisierung der Betroffenen leisten soll. „Das Projekt hat bei mir großes Interesse für das Thema Bewegungsförderung bei Demenz geweckt. Als angehende Gesundheits- und Pflegemanagerin sehe ich es zukünftig als bedeutsame Aufgabe, mich in diesem Bereich zu engagieren und weitere gesundheitsförderliche Initiativen zu starten", meint Marina Hedenik in Vertretung für das studentische Entwickler-Team.

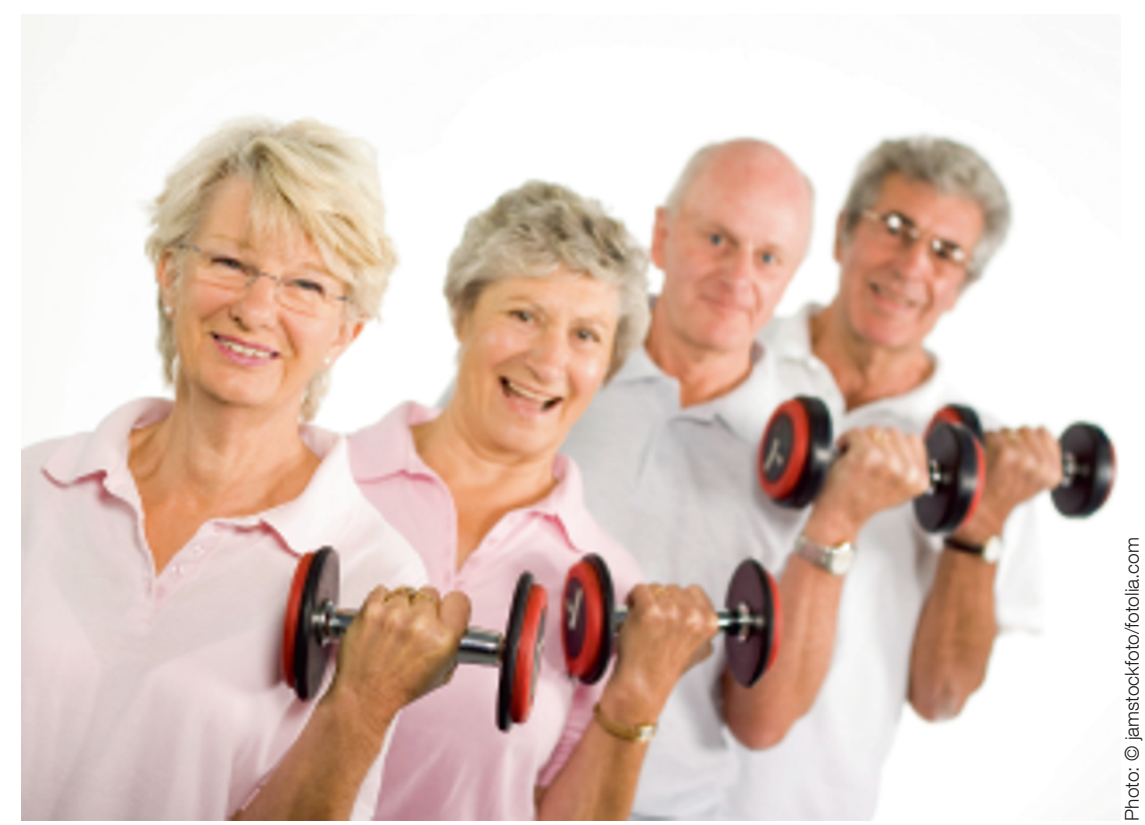

Bewegung wirkt sich auch förderlich auf die kognitiven Fähigkeiten im Alter aus.
Ganz in diesem Sinne werden in den nächsten Jahren weitere ambitionierte Projekte der Gesundheitsförderung von Studenten folgen. So liefern die Studiengänge Gesundheits- und Pflegemanagement der FH Kärnten bedeutsame Impulse für die Gesundheitsförderung unterschiedlicher Bevölkerungsgruppen und bieten den Studierenden die Gelegenheit, erste praktische Erfahrungen in der Gesundheitskommunikation zu sammeln.

Quelle: Presseinformation FH Kärnten

\section{Buchempfehlung}

\begin{tabular}{l} 
We bitte?" \\
Kommunikation in Gesundheitsberufen \\
Renate Tewes \\
$\begin{array}{l}\text { Heidelberg 2015, 2. Auflage } \\
138 \mathrm{~S} \text {. 16 Abb. in Farbe } \\
\text { Softcover } € 9,99 \text { (D) | } € 10,27(\mathrm{~A}) \mid \mathrm{sFr} 11.00(\mathrm{CH}) \\
\text { ISBN 978-3-662-46643-8 } \\
\text { Auch als eBook verfügbar }\end{array}$ \\
\hline
\end{tabular}

Reden Sie miteinander - aber richtig!

Das Buch „Wie bitte?“ aus der Reihe: „Top im Gesundheitsjob“ hilft Ihnen, Patienten und Mitarbeiter in Gesundheitsberufen besser zu verstehen. Nutzen Sie die Erfolgspotenziale gelungener Kommunikation.

Die Autorin Prof. Dr. Renate Tewes ist Krankenschwester, Diplom-Psychologin, Pflegewissenschaftlerin und arbeitet außerdem als Coach für Führungskräfte

- Kriterien einer guten Kommunikation für Team und Patienten

- Lösungsansätze bei Fehlinformationen und schlechtem Arbeitsklima

- Mit zahlreichen Beispielen, Übungen und Techniken

- Neu in der 2. Auflage: Individuell maßgeschneiderte Kommunikation 